\title{
Erratum to: Ecological, morphological and genetic diversity in Oryza rufipogon Griff. (Poaceae) from Hainan Island, China
}

\author{
Dong Yibo · Pei Xinwu • Yuan Qianhua • \\ Wu Hongjin • Wang Xujing · Jia Shirong • \\ Peng Yufa
}

Published online: 9 March 2010

(C) Springer Science+Business Media B.V. 2010

\section{Erratum to: Genet Resour Crop Evol DOI 10.1007/s10722-009-9523-2}

"Transgenic rice has been widely cultivated throughout Hainan Island without isolation (Dong et al. 2008; $\mathrm{Lu}$ et al. 2008). As a result, transgenic escape via gene flow from genetically modified cultivars to $O$. rufipogon has occurred." in the introduction was incorrectly published. It should be:

The online version of the original article can be found under doi:10.1007/s10722-009-9523-2.

D. Yibo · W. Hongjin · P. Yufa $(\bowtie)$

State Key Laboratory for Biology of Plant Disease and Insect Pests, Institute of Plant Protection, Chinese Academy of Agricultural Sciences, 100094 Beijing, China e-mail: Pyf@caascose.net.cn

P. Xinwu $(\bowtie) \cdot$ W. Xujing $\cdot$ J. Shirong Institute of Biotechnology, Chinese Academy of Agricultural Sciences, 100081 Beijing, China e-mail: Peixw@mail.caas.net.cn

Y. Qianhua

College of Agriculture Science, MOE Key Lab of Tropic Biological Resources, Hainan University, 570228 Haikou, China
"Recently, China has approved the insect-resistant Bt rice for commercial use, although 2-3 years of regional or nation-wide variety testing is still needed to be done. In accordance, the possible environment impact of transgene flow to common wild rice O. rufipogon should be monitored and assayed." 